ORIGINAL ARTICLE

\title{
Effectiveness of ultrasound screening for developmental dysplasia of the hip
}

\author{
E A Roovers, M M Boere-Boonekamp, R M Castelein, G A Zielhuis, T H Kerkhoff
}

Arch Dis Child Fetal Neonatal Ed 2005;90:F25-F30. doi: 10.1136/adc.2003.029496 See end of article for
authors' affiliations

Correspondence to: E A Roovers, Centre for Health Care Research, University of Twente, PO Box 217, Enschede 7500 $A E$, the Netherlands

Accepted 28 January 2004
Objective: To determine the effectiveness of ultrasound screening for developmental dysplasia of the hip (DDH) after the neonatal period.

Design: Prospective cohort study.

Setting: Child health care centres.

Participants: Infants attending the child health care centres.

Interventions: The intervention group ( $\mathrm{n}=5170$ ) was screened by ultrasound at 1,2 , and 3 months of age. The control group ( $n=2066$ ) was screened by routine physical examination as part of the programme for child health surveillance at the child health care centres ( $\mathrm{CHC}$ screening). For evaluation of the screening, the children in both the intervention and control group received an ultrasound examination after 6 months of age to detect any abnormality that might have been missed by the screening.

Results: The sensitivity of the ultrasound screening was $88.5 \%$, and the referral rate $7.6 \%$. As a result of the ultrasound screening, $4.6 \%$ of the children were treated. The sensitivity of the $\mathrm{CHC}$ screening was $76.4 \%$, with a referral rate of $19.2 \%$. The treatment rate was $2.7 \%$. Of the treated children in the ultrasound screening group, $67 \%$ were referred before the age of 13 weeks, whereas in the $\mathrm{CHC}$ screening group only $29 \%$ were referred before this age.

Conclusions: This study shows that ultrasound screening detects more children with DDH than $\mathrm{CHC}$ screening and that more of them are detected at an earlier age. To accomplish this, even fewer children have to be referred. However, even general ultrasound screening seems not to eradicate late cases of $\mathrm{DDH}$. The higher treatment rate in the population screened by ultrasound may be a result of overtreatment.
$\mathrm{E}$ arly diagnosis and treatment of developmental dysplasia of the hip (DDH) is considered to be very important for its prognosis and outcome. ${ }^{1}$ In 1962, both Von Rosen and Barlow published a screening test, called the Ortolani test and the Barlow test respectively, for the early detection of congenital dislocation of the hip in neonates. ${ }^{23}$ Since then, routine neonatal screening for $\mathrm{DDH}$ has been introduced world wide. ${ }^{4}$ Despite these neonatal screening programmes, late cases still arise. ${ }^{56}$

A more sensitive diagnostic test for DDH is ultrasonography, which was introduced by Graf in 1980. ${ }^{7}$ General neonatal ultrasound screening has been advocated and is nowadays a standard procedure in Germany, Austria, and Switzerland. ${ }^{8-10}$ In spite of the positive results reported, ultrasound screening has not yet been adopted in other countries because of the large costs of general ultrasound screening. These comprise the costs of organising and performing the screening (staff, facilities, and equipment) and the costs for additional diagnostic procedures. A second reason for rejecting ultrasound screening is the presumed risk of overtreatment when applied at a very young age. ${ }^{11-13}$ Postponing the screening beyond the neonatal period has been suggested to reduce the risk of overtreatment. Consensus on the best age for ultrasound screening has, however, not been reached. ${ }^{11} 1415$ Moreover, ultrasound screening after the neonatal period is difficult to implement in most countries because it is hard to ensure that all children are examined. ${ }^{15}$ In contrast, in the Netherlands, neonatal screening is difficult to achieve because of the large percentage of home deliveries. ${ }^{16}$ Instead, screening for DDH has become a part of the programme for child health surveillance, which has been organised by the child health care (CHC) centres. The CHC screening is based on identification of risk factors and repeated physical examination of the hip (abduction test and Galeazzi test) in children aged 15 months. As the validity of this screening programme was not satisfactory, we investigated whether ultrasound screening after the neonatal period yields better results. ${ }^{17}$ A large prospective cohort study, called the Soundchec study, was set up in the Netherlands in 1998 to investigate the value of ultrasound screening for DDH and to determine the best age at which to perform it. The children in the intervention population were screened by ultrasound examinations of the hip by Graf's method from the age of 1 month onwards. ${ }^{18}$ In this paper, the results of the screening programme as performed in the Soundchec study are compared with the results of standardised CHC screening for DDH as reported by Boere-Boonekamp et al in $1998 .{ }^{17}$

\section{MATERIALS AND METHODS Intervention population}

The intervention population comprised all children born in the catchment areas of two CHC organisations in the Eastern part of the Netherlands between 1 September 1998 and 30 November 1999 ( $n=6259$ ). Informed parental consent was obtained before participation in the study. The children were screened by an ultrasound examination of the hip at the age of 1, 2, and 3 months. For evaluation purposes, the children received an additional ultrasound examination at 8 months of age (reference test) to detect any abnormality that may have been missed by the ultrasound screening examinations or that developed after the last screening examination.

The ultrasound examinations were performed by radiology technicians using Graf's method during a special session at the CHC centre. ${ }^{18}$ The technicians were trained in hip

Abbreviations: $\mathrm{CHC}$, child health care; $\mathrm{DDH}$, developmental dysplasia of the hip 


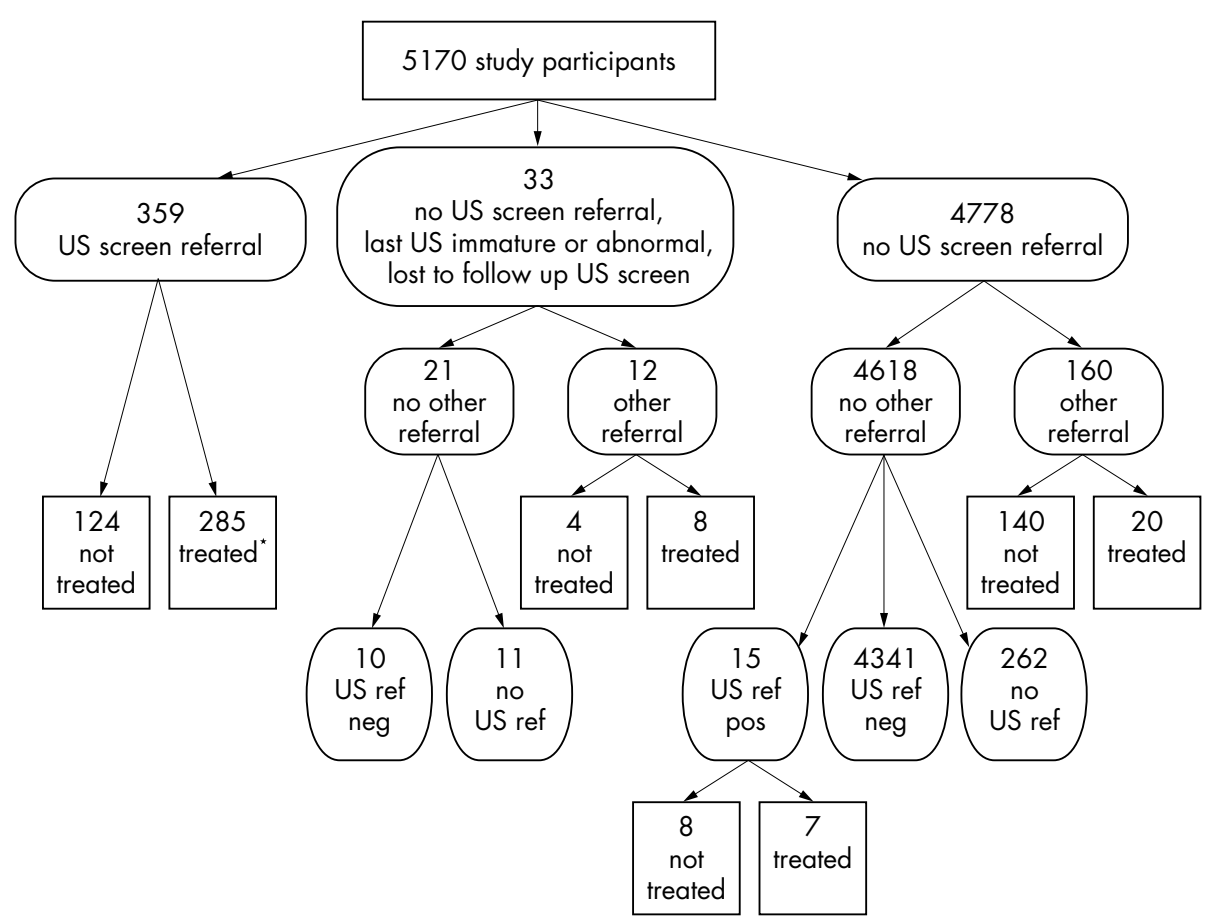

Figure 1 Schematic representation of the results of a post-neonatal ultrasound screening for developmental dysplasia of the hip with every child scheduled to undergo three ultrasound screening examinations in the first four months. *Four children were not treated until they were referred again at the ultrasound reference examination. US, Ultrasound; US ref, ultrasound reference examination; neg, negative; pos, positive.

sonography by Professor Graf or by the project team (radiologist, orthopaedic resident, orthopaedic surgeon, and CHC doctor) according to Graf's principles. The technicians performed the examinations under supervision of this project team. A portable sonograph with real time imaging was used for the ultrasound examinations. The apparatus (Hitachi EUB-405) was equipped with a linear array transducer operating on an ultrasound frequency of 7.5 or $5 \mathrm{MHz}$ (EUP-L33). A location device and a probe guiding device were used to standardise the infant positioning and scanning technique. ${ }^{19}$

At the first screening, only children with decentred hips (type D or worse) were referred. Severe immature (severe type IIa-) and abnormal hips (type IIb or worse) were indications for referral at the second and third screening. At the reference examination, all infants with abnormal results (type IIb or worse) were referred. The children who met the criteria were referred to a radiologist or an orthopaedic surgeon in the regional hospitals for diagnostic evaluation. In

Table 1 Sonographic results according to Graf's classification and treatment rate per hip type in 5170 children screened by ultrasound up to three times in the first 4 months of life

\begin{tabular}{lll}
\hline $\begin{array}{l}\text { Sonographic hip type } \\
\text { (worst affected hip) }\end{array}$ & Screening results & Children treated \\
\hline I (normal) & $3227(62.4)$ & $13(0.4)$ \\
Ila/lla+ (immature) & $1510(29.2)$ & $32(2.1)$ \\
Ila- (immature) & $144(2.8)$ & $42(29.2)$ \\
Ilb (abnormal) & $100(1.9)$ & $52(52.0)$ \\
Ilc (abnormal) & $38(0.7)$ & $20(52.6)$ \\
D (abnormal) & $113(2.2)$ & $84(74.3)$ \\
III (abnormal) & $37(0.7)$ & $26(70.3)$ \\
IV (abnormal) & $1(0.02)$ & $1(100.0)$ \\
Total & $5170(100)$ & $270(5.2)$ \\
\hline Values are $\mathrm{n}(\%)$. & &
\end{tabular}

addition to being screened by ultrasound, the children in the intervention group were also examined according to the Dutch standardised assessment protocol by the CHC doctor as part of the programme for child health surveillance; only children with a sustained abnormal physical examination were referred for further diagnostic work up. In the referred children, the diagnosis DDH was established by the radiologist or orthopaedic surgeon based on the outcome of additional diagnostic procedures.

The diagnostic evaluation usually consisted of a clinical and a radiological or sonographic examination of the hip. The choice between radiological examination using the criteria of Tönnis and Brunken ${ }^{20}$ and ultrasound using Graf's method ${ }^{18}$ depended on the expertise of the doctor involved. Information on these diagnostic procedures, diagnosis, treatment, and outcome of treatment was obtained by careful examination of the medical records after parents had given their permission.

\section{Control population}

The control population comprised all children born in the catchment area of a CHC organisation in the eastern part of the Netherlands between 1 November 1992 and 31 December $1993(\mathrm{n}=2105) \cdot{ }^{17}$ The children were screened for DDH according to a standardised assessment protocol, which is the routine screening programme for $\mathrm{DDH}$, and received an ultrasound reference examination at the age of 6 months for the purpose of evaluation. The CHC screening is based on identification of risk factors and repeated physical examination of the hip. The risk factors considered are a positive family history of DDH in first or second degree relatives, a breech presentation or specific abnormalities at physical examination (torticollis, foot abnormalities, neurological disorders). The physical examination of the hip consists of the abduction test and the Galeazzi test. Limited abduction is a clinical manifestation of a contracture of the adductor muscles, which is often present in dislocated and dysplastic hips. The Galeazzi sign—that is, a difference in knee 

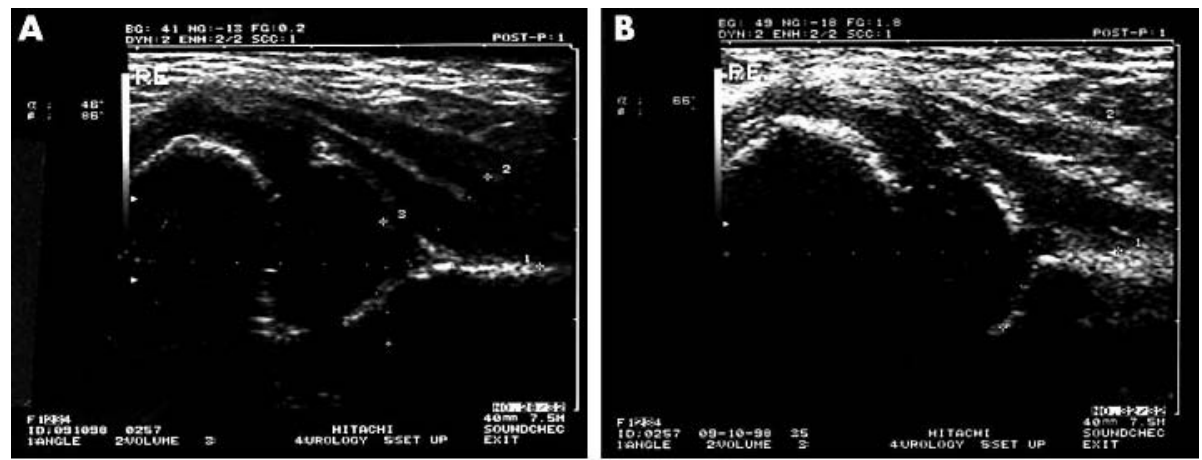

Figure 2 Sonograms of two nontreated children with type $D$ hips at the age of 5 weeks (A and C) and normal hips at the age of 35 and 32 weeks respectively ( $B$ and $D)$.
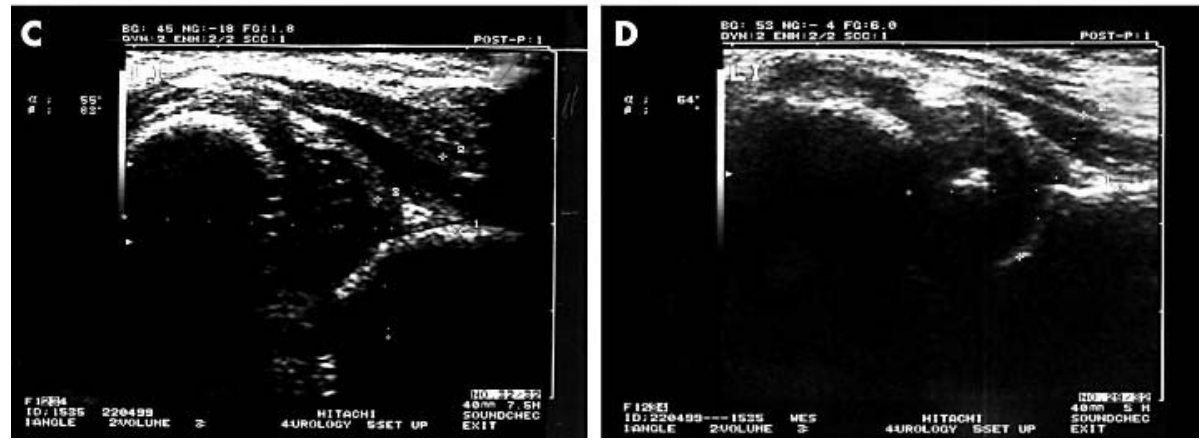

height-is an indication of apparent femoral shortening due to unilateral hip dislocation. ${ }^{1}$ Information on risk factors, physical examination, and referrals was registered after parental consent was obtained.

\section{Statistical analysis}

Differences in prevalence of risk factors between the two populations were tested using $\chi^{2}$ tests. Effectiveness of both screening programmes was analysed by a two by three table instead of the classical two by two table. ${ }^{21}$ In this two by three table, cases of DDH were divided into those diagnosed as a direct consequence of an abnormal finding in the screening programme and those diagnosed otherwise. Children who screened positive but whose DDH was nevertheless not detected at that time, but later on, actually did not benefit from the screening. Sensitivity was therefore defined as the screen positive children with screen detected DDH divided by all children with DDH. Other variables on which the screening programmes were evaluated were the referral rate, the age at referral, the treatment rate, the percentage of late detected cases, and the percentage of inpatient treatments.

\section{RESULTS}

In the unselected birth cohorts of the intervention and control population, the parents of 5170 children (82.6\%) and
$2066(98.1 \%)$ respectively agreed to participate. The intervention group had a slightly higher risk of DDH; significantly more infants in the intervention group were firstborn children $(47.4 \% v 41.1 \%)$ and had affected relatives both in the first or second degree $(11.2 \% v 7.6 \%)$ and in the third or fourth degree ( $13.6 \% \vee 10.2 \%)$ compared with the infants in the control group. There was no significant difference between the two groups with regard to sex, breech position, abnormalities of the feet, torticollis, and neurological disorders.

\section{Ultrasound screening}

In total, 5170 children participated in the ultrasound screening programme and were screened 2.7 times on average. Figure $\mathrm{l}$ is a schematic representation of the results. An abnormal ultrasound examination (type IIb or worse) on one or more occasions was found in 289 children (table 1). Of these children, 271 were referred for further diagnostic evaluation. In addition, 65 children with type IIa- hips, 20 children with type IIa hips, and three children with normal but suspect hips were referred for further diagnostic evaluation. Of these 359 referred children, 231 were subsequently treated for DDH. Another four children were initially judged not to require treatment, but were treated for DDH after an abnormal finding in the ultrasound reference

Table 2 Results of the ultrasound and child health care $(\mathrm{CHC})$ screening for developmental dysplasia of the hip (DDH) in modified two by two tables

\begin{tabular}{lllll}
\hline & \multicolumn{2}{l}{ DDH+ } & & \\
\cline { 2 - 3 } & Screen detected & Not screen detected & DDH - & Total \\
\hline Ultrasound screening + & 239 & 4 & 149 & 392 \\
Ultrasound screening - & 0 & 27 & 4751 & 4778 \\
Total & 239 & 31 & 4900 & 5170 \\
& & 7 & 335 & 397 \\
CHC screening + & 55 & 10 & 1659 & 1669 \\
CHC screening - & 0 & 27 & 1994 & 2066 \\
Total & 55 & & & \\
\hline
\end{tabular}


examination (type IIb) at the age of 8 months. The sonographic hip types of these four children in the screening were type IIa-, type IIb, and type D. Pelvic radiographs, produced at the age of at least 3 months, were assessed as normal. In three cases there appeared to be a discrepancy between the radiograph and the ultrasound (borderline cases). In retrospect, the radiograph of the fourth case had erroneously been classified as normal.

Thirty three children with an immature or abnormal ultrasound examination who were not referred at that time failed to complete the screening programme. Of these, eight were treated after a referral by the CHC doctor.

Of the children with normal ultrasound screening examinations, 27 were treated for DDH. The hips of these children were normal on all screening examinations or were immature (type IIa or type IIa-) at first, but turned out to be normal (type I) on subsequent screening examinations. The children were referred because of an abnormal ultrasound reference examination ( $\mathrm{n}=7$ ), an abnormal physical examination at the CHC centre $(\mathrm{n}=19)$, or a positive family history of $\mathrm{DDH}$ $(n=1)$. Most of these children were treated because the hip was dysplastic on pelvic radiographs. Because it is uncommon for sonographically normal hips to deteriorate, a panel of one radiologist and two orthopaedic surgeons reviewed the radiographs of 19 of these children. They judged 13 as being normal, five as abnormal, and one as not assessable.

All 4900 children who were not treated for DDH were scheduled to have an ultrasound examination at the age of 8 months to check for hip abnormalities missed by ultrasound screening. This examination resulted in seven additional cases being detected. The negative status was confirmed by the ultrasound reference examination in $93.6 \%$ of the 4900 children, and diagnostic evaluation in the hospital was negative in another $0.8 \%$. The reference examination confirmed that sonographically abnormal hips of children who were referred but not treated did actually resolve without treatment. Figure 2 gives an illustration of two of these cases. Although the parents were reminded of the reference examination, 273 children $(5.6 \%)$ were lost to follow up (fig 1). In total, 270 children were treated for DDH. Of these children, 231 were detected by the ultrasound screening and were treated early. Eight children who did not complete the screening programme were also treated after a referral by the CHC doctor. We counted them as screen detected cases because their hips were not normal at the last screening examination. Twenty seven children treated for DDH were not detected by the screening. Four children were treated after a referral at the ultrasound reference examination, although they had been referred before. They were considered as missed cases because they were not diagnosed as a consequence of the screening. Therefore the sensitivity of the ultrasound screening programme in detecting cases with DDH early was $88.5 \%$ (239/270, 95\% confidence interval $84.1 \%$ to $92.1 \%$ ) with a referral rate of $7.6 \%$ (tables 2 and 3 ). The percentage of children treated early was $4.6 \%$. Inpatient

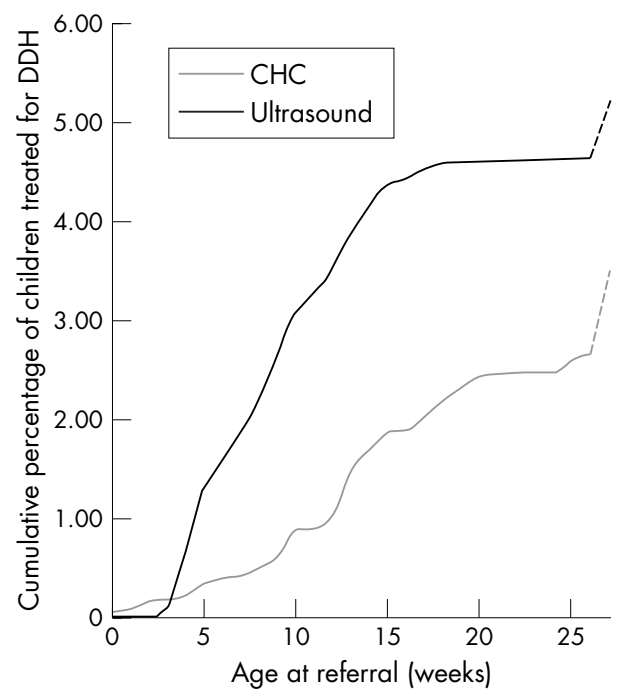

Figure 3 Age at referral of treated children participating in the ultrasound and child health care $(\mathrm{CHC})$ screening. DDH, Developmental dysplasia of the hip. Dotted line: treated children who had not been detected by screening.

treatment was seen in less than one child per 1000 screened children. The median age of the children at hospital admission was 25 weeks (range 14-27).

\section{CHC screening}

In total, 2066 children attended the $\mathrm{CHC}$ centre to participate in the screening for $\mathrm{DDH}$. On average, the children were examined 3.6 times by the $\mathrm{CHC}$ doctor. Of all the children, $19.2 \%$ were referred because of a positive family history of $\mathrm{DDH}$ in first or second degree relatives, a breech position during the last trimester of pregnancy, an abnormal finding on the physical examination of the hip, or because of other abnormalities (feet, torticollis, neurological disorders). In 55 of these children, the diagnosis was confirmed immediately. The hips of seven children were first judged to be normal, but they were treated for DDH after a referral at the ultrasound reference examination. In addition, 10 out of the 1669 screen negative children were treated after an abnormal finding on the ultrasound reference examination. Of the 1994 children who were not treated, $91.4 \%$ had a negative ultrasound reference examination, $3.7 \%$ had a negative examination in the hospital, and $4.9 \%$ were lost to follow up.

The sensitivity of the CHC screening to detect children with DDH early was $76.4 \%$ (55/72, 95\% confidence interval $64.9 \%$ to $85.6 \%$ ) (tables 2 and 3). The percentage of children treated early was $2.7 \%$. Three per 1000 screened children were admitted to hospital for DDH; the median age was 19 weeks (range 15-43).

\begin{tabular}{|c|c|c|c|c|}
\hline & $\begin{array}{l}\text { Ultrasound } \\
\text { screening } \\
(n=5170)\end{array}$ & $95 \% \mathrm{Cl}$ & $\begin{array}{l}\text { CHC screening } \\
(n=2066)\end{array}$ & $95 \% \mathrm{Cl}$ \\
\hline $\begin{array}{l}\text { Sensitivity (\%) } \\
\text { Referred children (\%) } \\
\text { Children treated early (\%) } \\
\text { Late detected cases (\%) } \\
\text { Inpatient treatment per } 1000 \text { screened } \\
\text { children }\end{array}$ & $\begin{array}{l}88.5 \\
7.6 \\
4.6 \\
0.6 \\
1\end{array}$ & $\begin{array}{l}84.1 \text { to } 92.1 \\
6.9 \text { to } 8.3 \\
4.1 \text { to } 5.2 \\
0.4 \text { to } 0.9 \\
0 \text { to } 2\end{array}$ & $\begin{array}{l}76.4 \\
19.2 \\
2.7 \\
0.8 \\
3\end{array}$ & $\begin{array}{l}64.9 \text { to } 85.6 \\
17.5 \text { to } 21.0 \\
2.0 \text { to } 3.5 \\
0.6 \text { to } 1.3 \\
1 \text { to } 7\end{array}$ \\
\hline
\end{tabular}




\section{Comparison of ultrasound and $\mathrm{CHC}$ screening}

Table 3 summarises the performance of both screening programmes for DDH and the consequences of the programmes. As significantly more children in the intervention group were firstborn children and had affected relatives compared with the children in the control group, the treatment rate was expected to be higher in the intervention group. Indirect standardisation of these risk factors showed that the expected treatment rate in the intervention group (including late cases) was 3.8\% instead of 3.5\%.

When the ages at referral are compared, another difference between the two screening programmes can be seen (fig 3): the cases in the ultrasound screening group were referred at an earlier age than those in the CHC screening group. The children in the ultrasound screening group can therefore be treated earlier, which may result in shorter treatment and better results. That the ultrasound screening resulted in a higher percentage of children being treated is also evident.

\section{DISCUSSION}

The sensitivity of the ultrasound screening programme in the Soundchec study was $88.5 \%$ compared with $76.4 \%$ for the routine $\mathrm{CHC}$ screening programme in the Netherlands. Remarkably, this higher sensitivity went together with a decrease in referral rate from $19.2 \%$ to $7.6 \%$. In addition, $67 \%$ of cases in the ultrasound screening group were referred before the age of 13 weeks. This is much higher than the $29 \%$ of cases in the CHC group referred before the age of 13 weeks.

It was disappointing that $11.5 \%$ of the cases of DDH-that is, six cases per 1000 screened children-were not detected by ultrasound screening, but only by active follow up of all children at the CHC centres or by the ultrasound reference examination at the age of 8 months. However, review of the diagnostic radiographs of these missed cases question the reliability of this finding. If almost $70 \%$ of the missed cases were not true cases of DDH, the sensitivity of the ultrasound screening would be $95 \%$ and the rate of missed cases 2.5 per 1000 children screened. Rosendahl et $a^{22}$ also reported the occurrence of late cases. Their rate was lower, with 1.4 late cases per 1000 screened children. This is an interesting phenomenon, all the more because their screening programme was confined to one ultrasound examination with repeat screening of only immature hips. Lewis $e t a^{23}$ did not detect any late cases in their population screened by ultrasound. A drawback of both studies is, however, the lack of active follow up of all screened children. Therefore it is most likely that not all late cases of DDH have been detected, especially those of dysplastic hips without dislocation.

It has largely been accepted that neonatal ultrasound screening carries the risk of overtreatment because some of the neonatal sonographically abnormal hips resolve without treatment. ${ }^{112425}$ For that reason, the children in the Soundchec study were first screened at the age of 1 month. Nevertheless, the treatment rate in the intervention group was high compared with that in the control group. In both the intervention and control group, an ultrasound reference examination at or after the age of 6 months was performed. Therefore one would not expect to explain the difference in treatment rate by the fact that not all cases of DDH were detected in the control group. The slightly higher risk of the children in the intervention group because of selective participation also could not account for the difference in treatment rate between the two groups. Therefore the difference in treatment rate is most likely explained by some degree of overtreatment in the population screened by ultrasound. This means that some of the abnormal hips that were treated would have normalised if left untreated. However, at the time treatment was initiated, these two groups could not be distinguished. Postponing the screening to the third or fourth month may decrease the risk of overtreatment, but it will have a negative effect on the age at referral, and therefore on the age at the start of treatment. Accepting the idea of overtreatment puts the sensitivity of ultrasound screening into perspective because overtreatment leads to overestimation of the sensitivity. However, correction for overtreatment resulted in a sensitivity of $84.2 \%$, which is still high compared with the sensitivity of the CHC screening programme.

Inpatient treatment (primarily traction) was more often indicated in the control population than the population screened by ultrasound. However, it is unclear whether this difference is a consequence of the ultrasound screening or a consequence of the treatment protocol, which has been changed; in 1993 most dislocations were treated by traction, in 1998 the Pavlik harness was usually the treatment of choice. As a result, in the intervention group, traction was started after abduction treatment had failed, whereas in the control group, traction was applied immediately after the diagnosis had been made (except for one child).

In conclusion, this study shows that ultrasound screening detects more children with DDH than CHC screening and that more of them are detected at an earlier age. To accomplish this, even fewer children have to be referred. However, general ultrasound screening seems not to eradicate late cases of $\mathrm{DDH}$. The higher treatment rate in the population screened by ultrasound points to overtreatment as a consequence of the screening. Prevention of overtreatment may be accomplished by performing the screening at the age of 2 or 3 months or by applying a referral protocol with a wait and see period for at least the immature hips.

\section{ACKNOWLEDGEMENTS}

Financial support was provided by the Netherlands Organisation for Health Research and Development (ZonMw).

\section{Authors' affiliations}

E A Roovers, M M Boere-Boonekamp, T H Kerkhoff, University of Twente, Enschede, the Netherlands

R M Castelein, University Medical Center, Utrecht, the Netherlands G A Zielhuis, University Medical Centre, Nijmegen, the Netherlands Competing interests: none declared

\section{REFERENCES}

1 Tönnis D. Congenital dysplasia and dislocation of the hip in children and adults. Berlin Heidelberg: Springer-Verlag, 1987

2 von Rosen S. Diagnosis and treatment of congenital dislocation of the hip joint in the newborn. J Bone Joint Surg [Br] 1962;44:284-91.

3 Barlow TG. Early diagnosis and treatment of congenital dislocation of the hip. $J$ Bone Joint Surg [Br] 1962;44:292-301.

4 Boere-Boonekamp MM, Verkerk PH. Screening for developmental dysplasia of the hip. Semin Neonatol 1998;3:49-59.

5 Leck I. An epidemiological assessment of neonatal screening for dislocation of the hip. J R Coll Physicians Lond 1986;20:56-62.

6 Clarke NM. Diagnosing congenital dislocation of the hip. BM 1992;305:435-6.

7 Graf $\mathbf{R}$. The diagnosis of congenital hip-joint dislocation by the ultrasonic Compound treatment. Arch Orthop Trauma Surg 1980;97:117-33.

8 Bon RA, Exner GU. Fruhdiagnose der Huftdysplasie: Argumente fur ein generelles sonographisches Screening in der Schweiz. Schweiz Rundsch Med Prax 1992;81:519-23.

9 Graf R, Tschauner C, Klapsch W. Progress in prevention of late developmental dislocation of the hip by sonographic newborn hip "screening": results of a comparative follow-up study. J Pediatr Orthop B 1993;2:115-21.

10 Gunther KP, Stoll S, Schmitz A, et al. Erste Ergebnisse aus der Evaluationsstudie des sonographischen Huftscreenings in der Bundesrepublik Deutschland. Z Orthop Ihre Grenzgeb 1998;136:508-12.

11 Harcke HT, Kumar SJ. The role of ultrasound in the diagnosis and management of congenital dislocation and dysplasia of the hip. J Bone Joint Surg [Am] 1991;73:622-8.

12 Hansson G, Jacobsen S. Ultrasonography screening for developmental dysplasia of the hip joint. Acta Paediatr 1997;86:913-15

13 Clinical practice guideline: early detection of developmental dysplasia of the hip. Committee on Quality Improvement, Subcommittee on Developmental 
Dysplasia of the Hip. American Academy of Pediatrics. Pediatrics 2000; 105:896-905

14 Donaldson JS, Feinstein KA. Imaging of developmental dysplasia of the hip. Pediatr Clin North Am 1997:44:591-614.

15 Wientroub S, Grill F. Ultrasonography in developmental dysplasia of the hip. J Bone Joint Surg [Am] 2000;82:1004-18.

16 Castelein RM. Fysische diagnostiek-de handgreep van Ortolani. Ned Tijdschr Geneeskd 2002;146:1077-80.

17 Boere-Boonekamp MM, Kerkhoff TH, Schuil PB, et al. Early detection of developmental dysplasia of the hip in the Netherlands: the validity of a standardized assessment protocol in infants. Am J Public Health 1998;88:285-8.

18 Graf R. Kursus der Hüftsonographie beim Säugling. Stuttgart: Gustav Fischer Verlag, 1995.

19 Muller W, Lercher K, DeVaney TT, et al. Untersuchungsfehler durch Schallkopfkippung bei der Huftsonographie nach Graf. Ultraschall Med 2001;22:48-54.
20 Tönnis D, Brunken D. Eine Abgrenzung normaler und pathologischer Huftpfannendachwinkel zur Diagnose der Huftdysplasie. Auswertungen von 2294 Pfannendachwinkeln kindlicher Huftgelenke. Arch Orthop Unfallchir 1968;64:197-228.

21 Grimes DA, Schulz KF. Uses and abuses of screening tests. Lancet 2002;359:881-4

22 Rosendahl K, Markestad T, Lie RT. Ultrasound screening for developmental dysplasia of the hip in the neonate: the effect on treatment rate and prevalence of late cases. Pediatrics 1994:94:47-52.

23 Lewis K, Jones DA, Powell N. Ultrasound and neonatal hip screening: the fiveyear results of a prospective study in high-risk babies. J Pediatr Orthop 1999; 19:760-2.

24 Catterall A. The early diagnosis of congenital dislocation of the hip. J Bone Joint Surg [Br] 1994;76:515-16.

25 Bialik V, Bialik GM, Wiener F. Prevention of overtreatment of neonatal hip dysplasia by the use of ultrasonography. J Pediatr Orthop B 1998;7:39-42.

\section{Call for Book Reviewers}

Book reviews are a popular feature of $A D C$, and many readers use them to decide how best to spend scarce library funds. We need to recruit a team of willing reviewers, both generalists and specialists, who are prepared to read and review new books (and CD-ROMs, etc) within a three-month deadline: could you help? You will have the option to decline if you can't manage a review in time.

Unfortunately $A D C$ cannot pay reviewers, but you will be able to keep the book for yourself or your department. Trainees are particularly welcome to apply.

For logistical reasons reviewers should be based in the UK or Republic of Ireland and internet access is essential.

Please contact archdischild@bmigroup.com with brief details of special interests and reviewing experience, if any (include BOOK REVIEWS in the subject field). 